\title{
Hybridization of Stochastic Local Search and Genetic Algorithm for Human Resource Planning Management
}

\author{
Andrej Škraba', Vladimir Stanovov ${ }^{2}$, Eugene Semenkin ${ }^{2}$, Davorin Kofjač ${ }^{1}$ \\ ${ }^{1}$ University of Maribor, Faculty of Organizational Sciences, Kidričeva cesta 55a, SI-4000 Kranj, Slovenia \\ andrej.skraba@fov.uni-mb.si, davorin.kofjac@fov.uni-mb.si \\ ${ }^{2}$ Reshetnev Siberian State Aerospace University, Institute of Computer Science and Telecommunications, \\ 31 »Krasnoyarskiy Rabochiy« ave., Krasnoyarsk, 660037, Russian Federation \\ vladimirstanovov@yandex.ru, eugenesemenkin@yandex.ru
}

\begin{abstract}
Background and Purpose: The restructuring of human resources in an organization is addressed in this paper, because human resource planning is a crucial process in every organization. Here, a strict hierarchical structure of the organization is of concern here, for which a change in a particular class of the structure influences classes that follow it. Furthermore, a quick adaptation of the structure to the desired state is required, where oscillations in transitions between classes are not desired, because they slow down the process of adaptation. Therefore, optimization of such a structure is highly complex, and heuristic methods are needed to approach such problems to address them properly.

Design/Methodology/Approach: The hierarchical human resources structure is modeled according to the principles of System Dynamics. Optimization of the structure is performed with an algorithm that combines stochastic local search and genetic algorithms.

Results: The developed algorithm was tested on three scenarios; each scenario exhibits a different dynamic in achieving the desired state of the human resource structure. The results show that the developed algorithm has successfully optimized the model parameters to achieve the desired structure of human resources quickly.

Conclusion: We have presented the mathematical model and optimization algorithm to tackle the restructuring of human resources for strict hierarchical organizations. With the developed algorithm, we have successfully achieved the desired organizational structure in all three cases, without the undesired oscillations in the transitions between classes and in the shortest possible time.
\end{abstract}

Keywords: stochastic local search, system dynamics, human resources, manpower, simulation

\section{Introduction}

Human resource planning is one of the main activities in every organization that has a long-term mission plan. In our previous research (Škraba et al., 2011; Škraba et al., 2015a; Škraba et al., 2015b; ک̌kraba et al., 2015c; Kofjač et al., 2015), we have examined human resources planning in strict hierarchical organizational structures, such as the army, or other organizations, in which promotions to higher classes are possible only by one step at a time. The problem of the hierarchical manpower control is challenging due to the variability of the constraints on the recruitment, transitions, wastage, and retirements. Furthermore, the dynamics of the system should be considered, usually in a longer period, i.e. ten years or more. In order to provide proper strategies to restructure human resources, several techniques have been applied. For the prevention of oscillations, the finite automaton was developed (Škraba et al., 2011) combined

Received: $14^{\text {th }}$ August, 2015; revised: $5^{\text {th }}$ October 2015; accepted; $15^{\text {th }}$ November 2015 
with genetic algorithms and pattern search. In optimization methods, the self-configuring algorithms were developed (Škraba et al., 2015b) providing promising results. In addition to the problem of the oscillations, the variable parameter boundaries were considered (Škraba et al. 2015c).

Human resource planning affects the entire organization. In the research of Majd et al. (2015), the combination of human resource planning and production-inventory control is considered. The problem was addressed by the modelling and simulation methods providing promising results in comparison to other approaches. The importance of a hybrid simulation model tackling management problems was indicated in Kljajić et al. (2000). In this work, System Dynamics Methodology has be used (Škraba et al., 2003; Škraba et al., 2007; Borštnar et al., 2011; Rozman et al., 2013). A resource assignment language was developed in the research of Cabanillas et al., (2015), which provided the automatic answers to tackle the problem of the management of resources in business process at the design phase. In the study of Lin et al. (2015), the mixed integer programming model was used to solve the problem of workforce scheduling for retail stores, taking into account employee's preferences and the equality of scheduling. Here the goal was to achieve an optimal balance among employee's satisfaction, customer service level, and labor costs. Unsurprisingly, the human resource planning requires advanced quantitative methods to provide proper results. Gupta and Ghosal (2014) applied stochastic modelling for determination of the appropriate time of promotion considering the survival rates in different classes. They have considered varying size classes and indicated, that the main goal of an employee in an organization is to get a promotion to the next grade. Nevertheless, the main obstacles to the organizational restructuring regarding human resources management are in organizational barriers (Babaei et al., 2015). Technological factors should, therefore, be considered to be less important than the organizational obstacles.
Our goal is to provide the proper mathematical model of the strict hierarchical human resources model and an appropriate optimization algorithm, that would provide the transition strategies for particular classes, which would enable us to restructure the organization in the shortest possible time without undesired oscillations in the strategy.

\section{Methodology}

The structure of the organizational human resources system could be best described by the application of the Causal Loop Diagram (CLD). Figure 0 shows the structure of the system, in which $n$ classes are considered. Starting with Class 1 at the left, recruitment is increasing the number of employees in Class 1. If the number of members of Class 1 increases, the Promotions from Class 1 to Class 2 increases above the number that it would have otherwise been. In contract the other hand, if the Number of Promotions from Class 1 to Class 2 increases, the number in Class 1 is lower than it would have otherwise been. This forms the stabilizing, negative feedback loop, which is cascading throughout our structure. The amount of waste decreases the number of people in each particular class. The promotion factor determines the rate of promotions from lower classes to the higher classes. More promotions from Class 1 to Class 2 increase the number of people in Class 2, etc. At the end of the structure, retirement is considered to be the exit from the system. The structure consists of the cascaded structure of decay determining the dynamics of the system.

The system was modelled using the System Dynamics methodology (Sterman, 2000). In a compact form, the dynamics are described in the state space:

$$
\begin{aligned}
& \dot{\mathbf{x}}=\mathbf{A x}+\mathbf{B u} \\
& \mathbf{y}=\mathbf{C x}+\mathbf{D u}
\end{aligned}
$$

where $\mathbf{x}$ represents the state vector, $\mathbf{y}$ the output of the system, $\mathbf{A}$ is the matrix of coefficients, $\mathbf{B}$ is input matrix, $\mathbf{u}$,

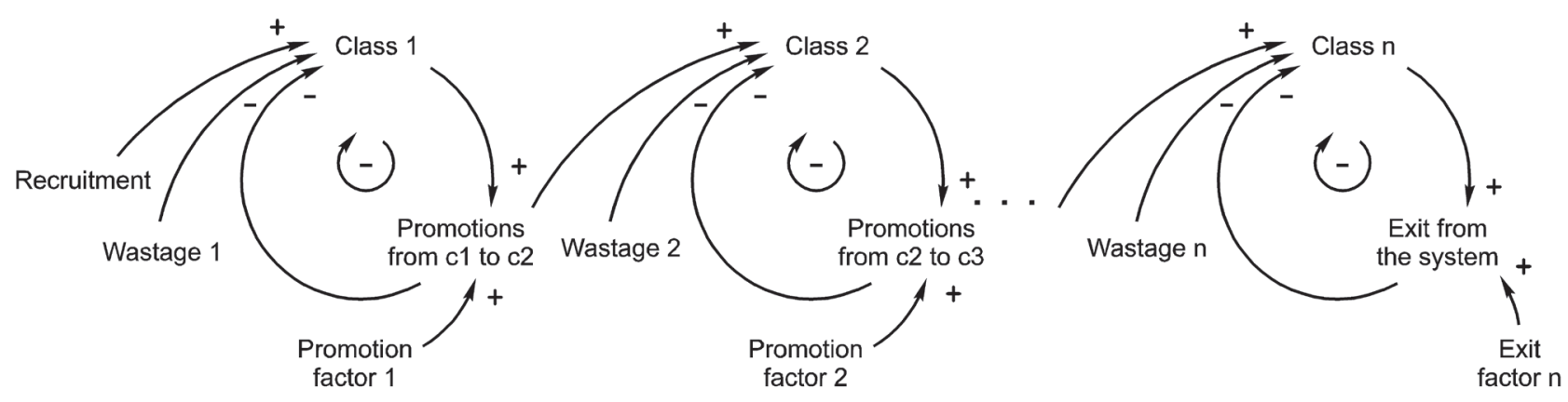

Figure 0: CLD of Organizational Human Resources 
vector of inputs, $\mathbf{C}$ output matrix and $\mathbf{D}$ translation matrix providing the input values to the desired output. Therefore, the system has been described as the set of the differential equations in the state space. A more detailed description could be found in the reference Škraba et al., 2015a.

The developed model provides us with the dynamic response. In order to obtain the appropriate strategies, that would enable achieving proper numbers of people in a particular class, the optimization algorithm was developed, which is applied to the model. In order to achieve the desired number of people in a particular rank, the goal is to determine how to define recruitment, transitions between ranks and fluctuations to reach a new, i.e. the desired, organizational structure.

The pseudocode of the optimization algorithm for finding transition values for one iteration is provided in the next section. The code should be launched several times until two last iterations are equal (i.e. the system is in equilibrium). The parameter $r$ represents promotions, $f$ factor of wastage, min and max represent parameter boundaries.

For all $r$ and $f$ set:

$$
\begin{aligned}
& r=\left(r_{\text {min }}+r_{\text {max }}\right) / 2 \\
& f=\left(f_{\text {min }}+f_{\text {max }}\right) / 2 \\
& \text { recruitment }=\text { max_recruitment } / 2
\end{aligned}
$$

For all classes calculate next iteration $X$

Repeat the following algorithm for $N$ steps:

For classes from 1 to NumberOfClasses:

If class number is 0 :

If current $X$ value is lower than desired:

Choose one of three actions randomly:

1) Decrease outflow by $l$ by changing $f$ value, if possible

2) Decrease promotion by 1 to the next class by changing $r$, if possible

3) Increase recruitment by 1 if possible

Else if current $X$ value is higher than desired:

Choose one of three actions randomly:

1) Increase outflow by $l$ by changing $f$ value, if possible

2) Increase promotion by 1 to the next class by changing $r$, if possible

3) Decrease recruitment by 1 if possible

For all classes recalculate next iteration $\mathrm{X}$

If class number is larger than 0 but lower than NumberOfClasses:

If current $X$ value is lower than desired:

Choose one of three actions randomly:

1) Decrease outflow by 1 by changing $f$ value, if possible

2) Decrease promotion by 1 to the next class by changing $r$, if possible

3 ) Increase promotion by 1 from previous class by changing $r$, if possible

Else if current $X$ value is higher than desired:

Choose one of three actions randomly:

1) Increase outflow by $l$ by changing $f$ value, if possible

2) Increase promotion by 1 to the next class by changing $r$, if possible

3) Decrease promotion by 1 from previous class by changing $r$, if possible

For all classes recalculate next iteration $X$

If class number is equal to NumberOfClasses:

If current $X$ value is lower than desired:

Choose one of three actions randomly:

1) Decrease outflow by 1 by changing $f$ value, if possible

2) Decrease output from the system by 1 by changing $r$, if possible

3 ) Increase promotion by 1 from previous class by changing $r$, if possible

Else if current $X$ value is higher than desired:

Choose one of three actions randomly:

1) Increase outflow by $l$ by changing $f$ value, if possible

2) Increase output from the system by 1 by changing $r$, if possible

3) Decrease promotion by 1 from previous class by changing $r$, if possible

For all classes recalculate next iteration $X$

Copy current $r, f$ and recruitment values to the next iteration. 
This algorithm allows finding the solution for the class problem using an iterative approach. The random choice of one of three different actions in each of the possible situations is because these actions influence not only the previous and next class nodes, but also all the nodes after the next. Because of the complexity of such dependencies, and the fact that a case with integer values is under consideration, the random choice of the next step was used.

Although this algorithm is capable of finding a solution, this solution depends on the initial values of the transitions, fluctuations, and recruitment. Because the numbers of transitions, fluctuations, and recruitment are quite large even for several classes, we used the genetic algorithm as an optimizing technique for this problem. The optimized values were the initial positions of the transition and fluctuation coefficients. Therefore, the proposed algorithm for finding the optimal strategy given the initial values can be considered to be the local search method. The fitness values for GA were calculated as the average number of steps required to achieve the goal position plus the penalty values.

The goal position was achieved only if all the class values were equal to the desired ones, and transition and fluctuation values are the same for two last time steps, as well as the number of people (this means that the system is stable/in the equilibrium point).

For every goal function calculation in GA, 100 runs of the proposed strategy search algorithm were used, and then used the minimal number of steps required to achieve the basic fitness value. Every time, we received a better solution, we saved it separately.

Together with the number of time steps, two more criteria were included into the goal function: the average recruitment level needed to achieve the goal and stabilize the system (normalized to the maximum recruitment number) with a weight of 0.001 as well as the penalty value, depending on the oscillations. The penalty values were calculated using a derivative-based approach (Škraba et al., 2015b). The initialization of the population of GA was performed randomly; the size of the population was 100 , and the number of generations was 1000 .

\section{Results}

A large organization restructuring problem with a strict hierarchical structure has been under consideration (Škraba et al., 2011). The developed algorithm was used to consider the three experiments that were made for the initial values presented in Table 1.
Table 1. Initial numbers of class members

\begin{tabular}{|l|c|c|}
\hline Class & State variable & [people] \\
\hline C1 & $x_{1}$ & 256 \\
\hline C2 & $x_{2}$ & 258 \\
\hline C3 & $x_{3}$ & 447 \\
\hline C4 & $x_{4}$ & 189 \\
\hline C5 & $x_{5}$ & 119 \\
\hline C6 & $x_{6}$ & 42 \\
\hline C7 & $x_{7}$ & 11 \\
\hline C8 & $x_{8}$ & 3 \\
\hline
\end{tabular}

Since the goal is to obtain the optimal transition and recruitment coefficients between particular classes, their lower and upper boundaries have to be considered; these are shown in Table 2 . Here $\bar{r}$ represents the average value of the promotion parameters, $\sigma_{r}$ is standard deviation, min and max represent maximum values for particular parameters; similar for wastages $f$.

Table 2: Transition coefficients and corresponding statistics

\begin{tabular}{|l|c|c|c|c|c|c|c|c|}
\hline Class & $\bar{r}$ & $\sigma_{r}$ & $r_{\min }$ & $r_{\max }$ & $\bar{f}$ & $\sigma_{f}$ & $f_{\min }$ & $f_{\max }$ \\
\hline $\mathrm{C} 1$ & 0.16 & 0.13 & 0.06 & 0.43 & 0.13 & 0.09 & 0.04 & 0.30 \\
\hline $\mathrm{C} 2$ & 0.12 & 0.09 & 0.04 & 0.32 & 0.11 & 0.06 & 0.06 & 0.24 \\
\hline $\mathrm{C} 3$ & 0.07 & 0.06 & 0.03 & 0.20 & 0.07 & 0.04 & 0.03 & 0.15 \\
\hline $\mathrm{C} 4$ & 0.10 & 0.10 & 0.03 & 0.33 & 0.09 & 0.06 & 0.02 & 0.20 \\
\hline $\mathrm{C} 5$ & 0.07 & 0.05 & 0.02 & 0.18 & 0.08 & 0.06 & 0.02 & 0.16 \\
\hline $\mathrm{C} 6$ & 0.05 & 0.04 & 0.01 & 0.11 & 0.15 & 0.10 & 0.05 & 0.37 \\
\hline $\mathrm{C} 7$ & 0.01 & 0.04 & 0.00 & 0.11 & 0.15 & 0.10 & 0.00 & 0.28 \\
\hline $\mathrm{C} 8$ & 0.01 & 0.05 & 0.00 & 0.10 & 0.15 & 0.10 & 0.00 & 0.30 \\
\hline
\end{tabular}

In Table 3, the three different goal positions are shown. The differences in these scenarios are the rate of reduction/ increase of people in particular classes. SC2 and SC3 are therefore scenarios with more demanding restructuring regarding the change in particular rank.

Table 3: Three scenarios used

\begin{tabular}{|l|c|c|c|}
\hline Class & SC1 & SC2 & SC3 \\
\hline C1 & 148 & 110 & 110 \\
\hline C2 & 289 & 304 & 304 \\
\hline C3 & 339 & 221 & 151 \\
\hline C4 & 224 & 165 & 109 \\
\hline C5 & 121 & 83 & 53 \\
\hline C6 & 45 & 32 & 13 \\
\hline C7 & 16 & 7 & 6 \\
\hline C8 & 6 & 5 & 3 \\
\hline
\end{tabular}


For the first scenario, the following solution was found, having three time steps. The fourth time step is needed to ensure that the system is stable. In Figure 1, the recruitment level into the first class is shown.

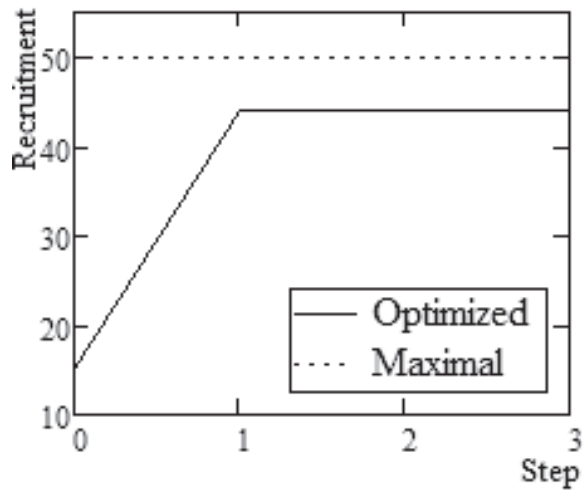

Figure 1. Recruitment level

In Figures 2 and 3, the change of the number of people in each class is shown. At time step 0 , the initial values are shown. At timestep 1, for most of the classes, the goal values are achieved.

\section{Class 1}

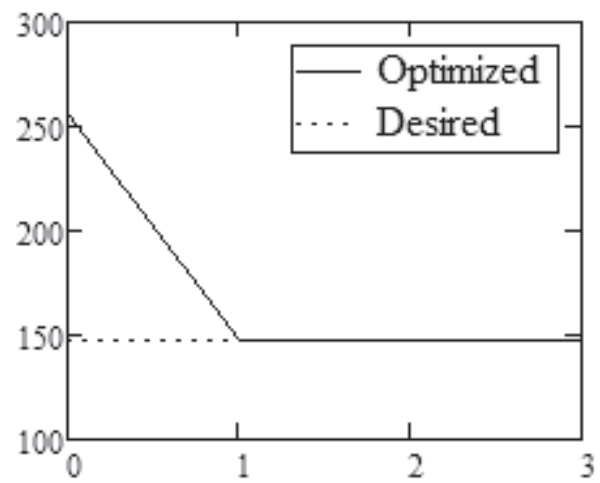

Class 2

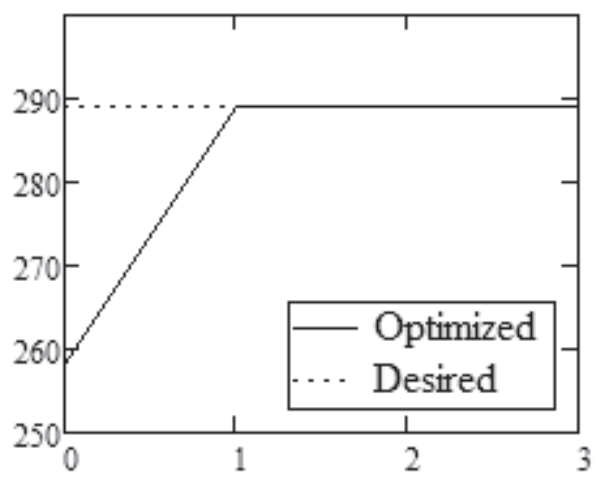

Class 3

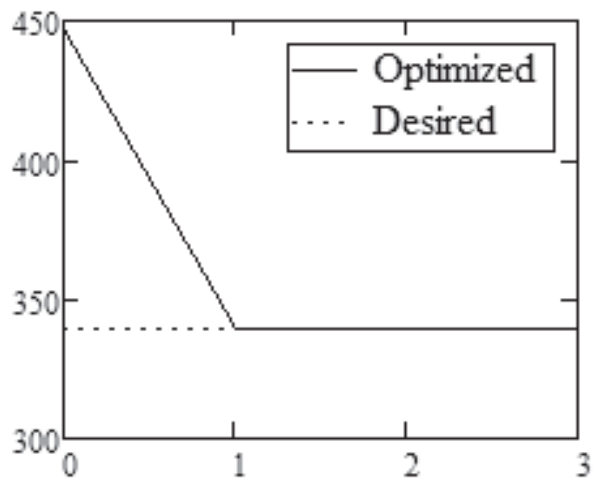

Class 4

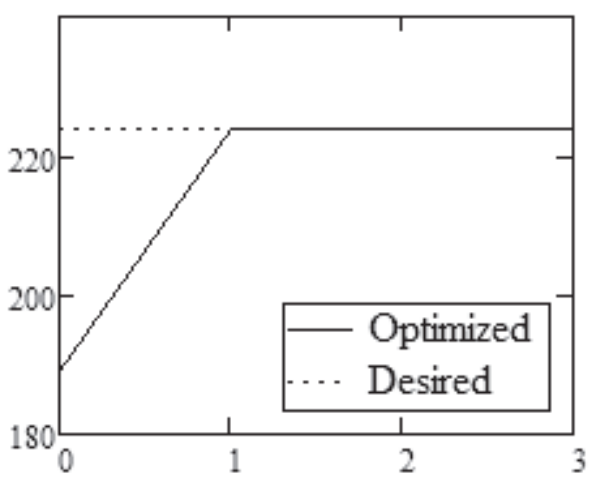

Figure 2. Classes 1-4

For classes 7 and 8 , it is not possible to achieve the goal in one step due to the transition coefficient boundaries so that the algorithm finds the best possible solution in two steps.

\section{Class 5}

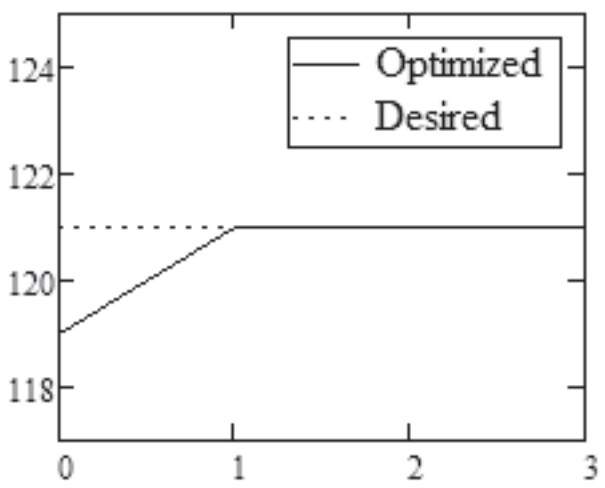




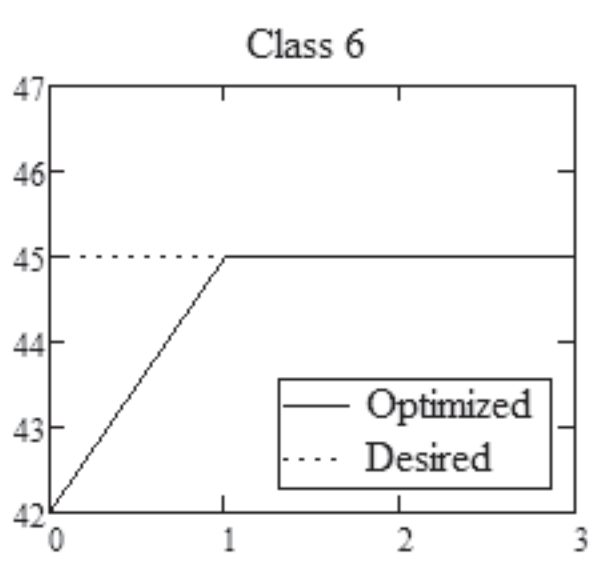

\section{Class 7}

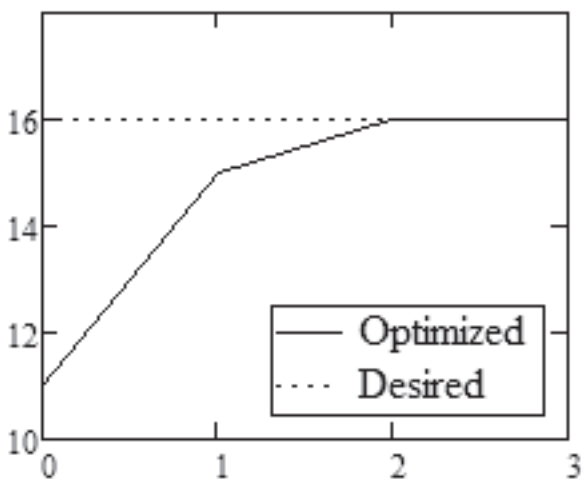

Class 8

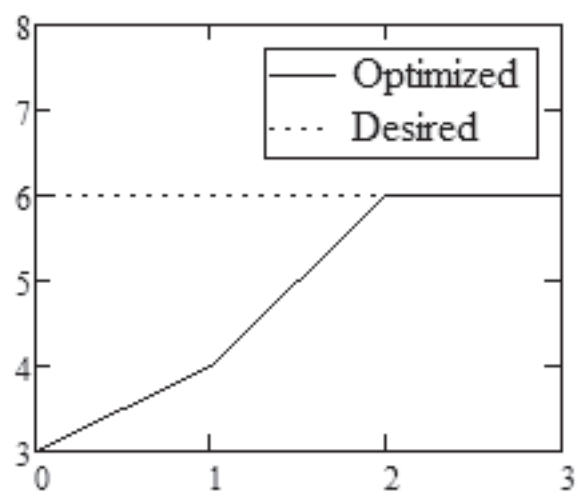

Figure 3. Classes 5-8

Figure 4 shows the transition and fluctuation coefficients achieved by the algorithm. Different colors represent transitions and fluctuations from particular classes.

\section{Transitions}

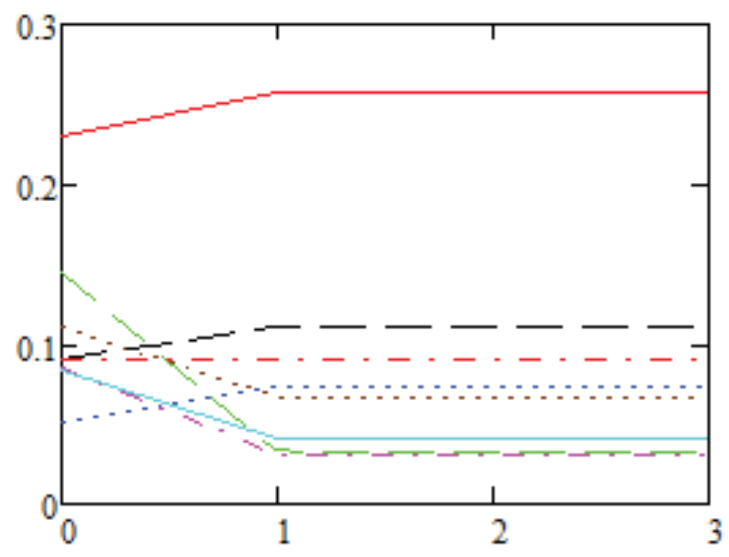

\section{Fluctuations}

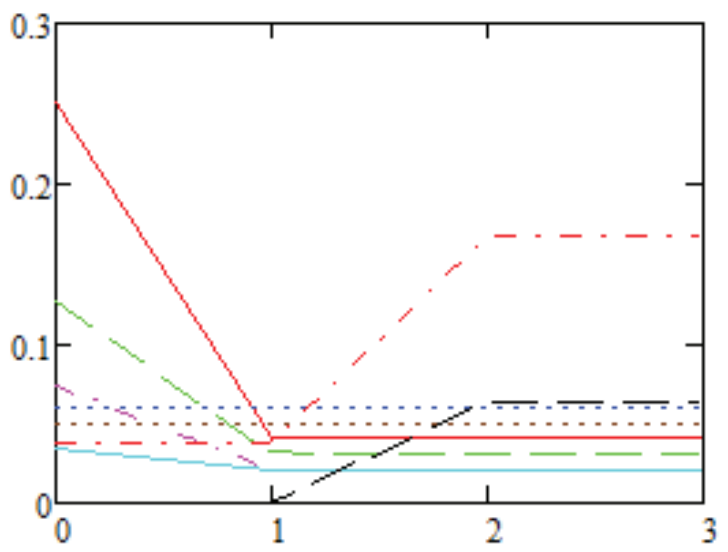

Figure 4. Transition and fluctuation coefficients

The last step shows that the transition and fluctuation values are stable, as are the number of people in each class. In Figure 5, the values of transitions and fluctuations are presented.

\section{Transitions}

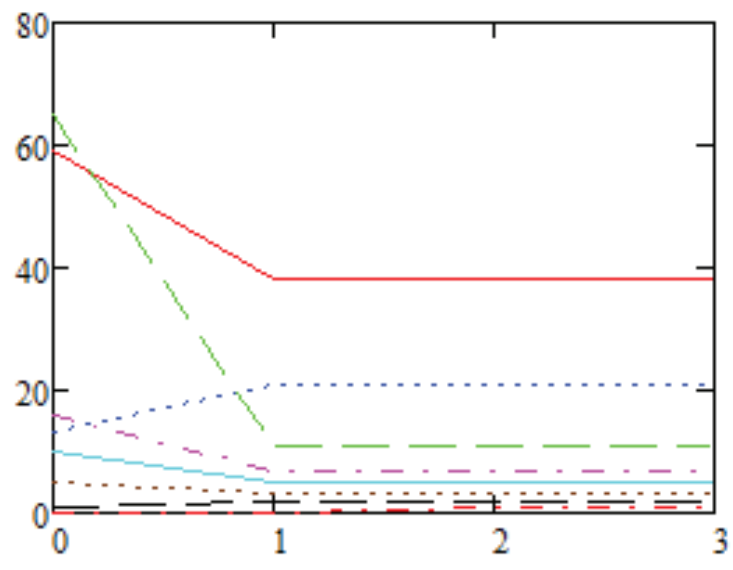


Fluctuations

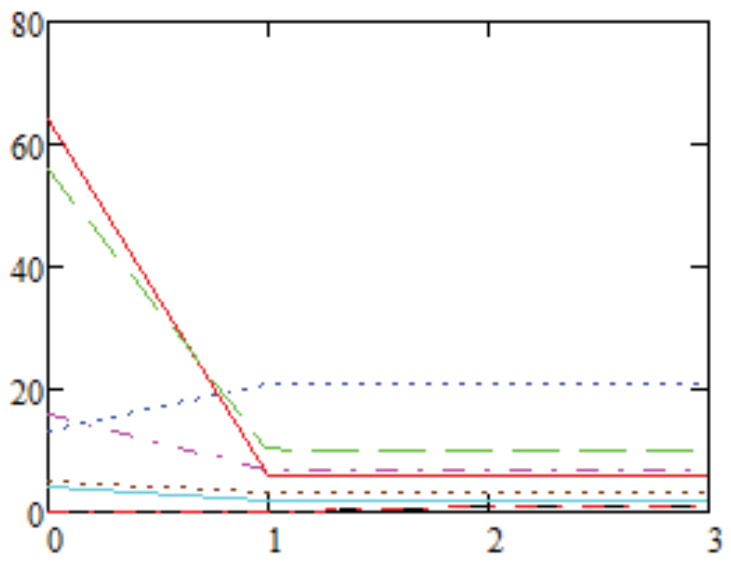

Figure 5. Transition and fluctuation values

From the presented figures, for this scenario, it may be concluded that the proposed approach enabled finding the solution without oscillations and in the least possible number of steps. This solution is not the only possible one, as the transition and fluctuation coefficients can be changed in each class while maintaining the system in a state of equilibrium.

For the second scenario, the following results were achieved. The recruitment level into the first class change is shown in Figure 6. For this case, the algorithm requires six steps to achieving the goal.

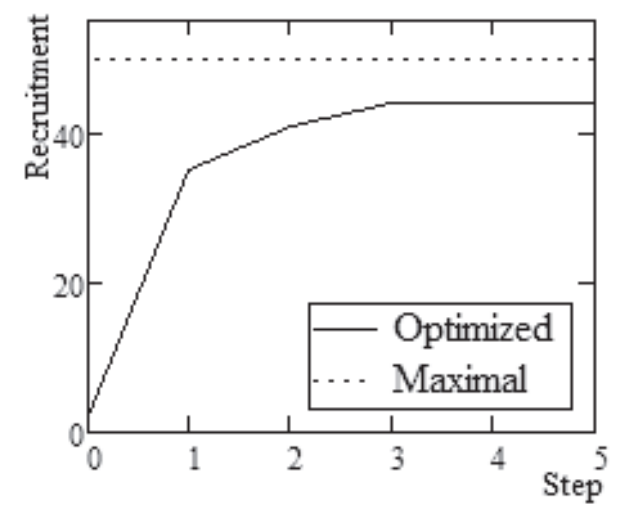

Figure 6. Recruitment level, second scenario

The recruitment level gradually rises but does not achieve the maximum possible level. In Figures 7 and 8, the change of the number of people in each class is shown. At time step 0 , the initial values are shown. For classes 1 and 2 , the goal is achieved in one step, while the other classes require more steps. For most classes the goal is not achievable in one step because of the constraints. In this case, the algorithm moves the current values of classes towards the goal and proceeds to the next step.

\section{Class 1}

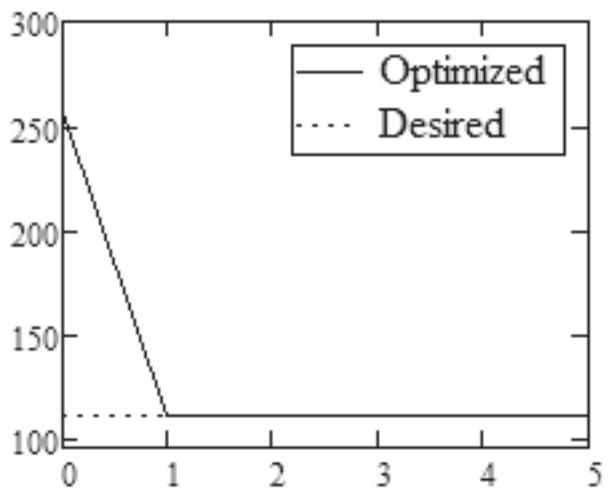

Class 2

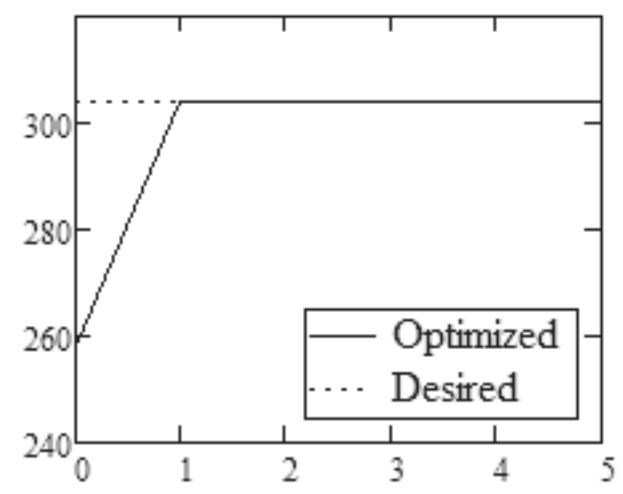

Class 3

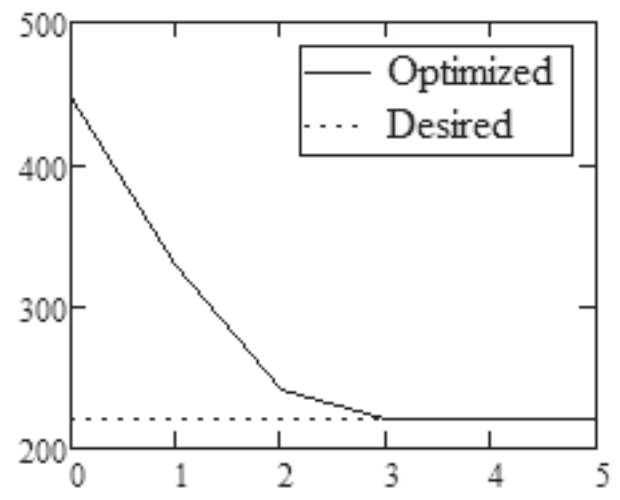




\section{Class 4}

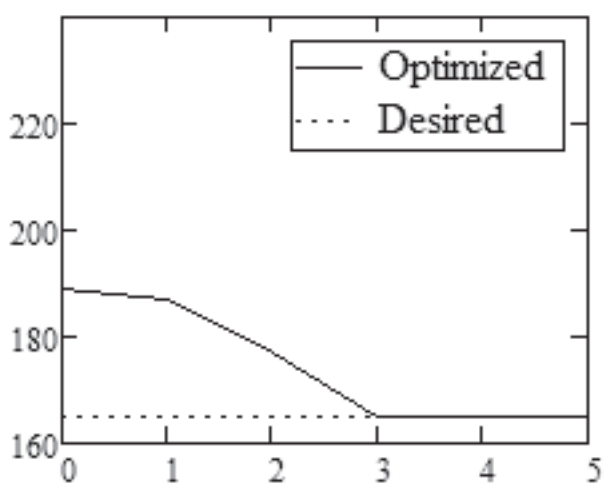

Figure 7. Classes 1-4, second scenario

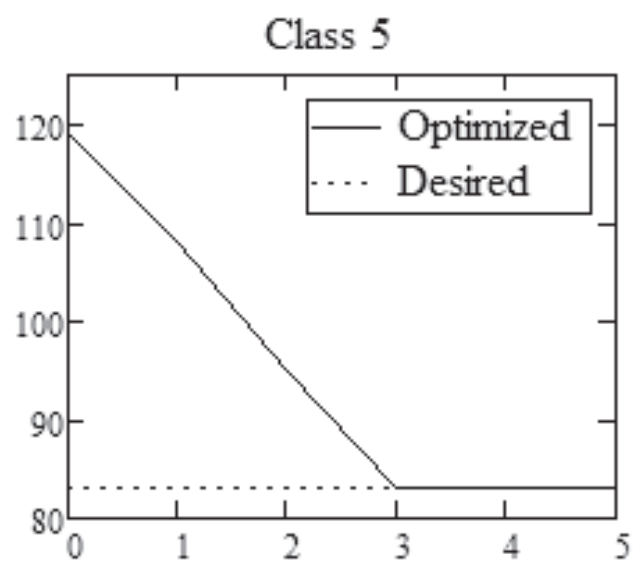

\section{Class 6}

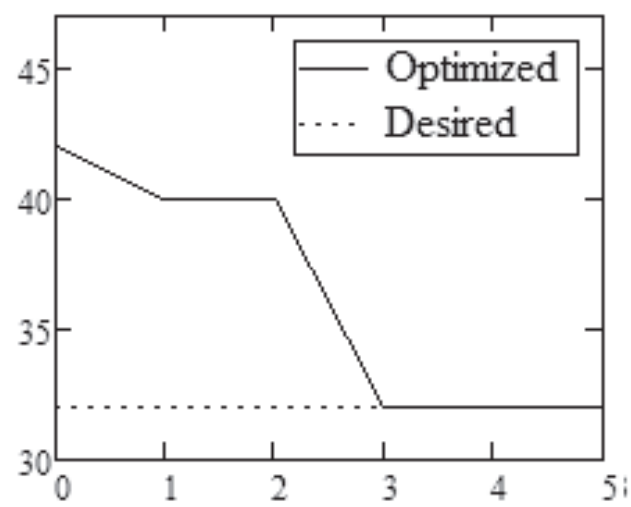

\section{Class 7}

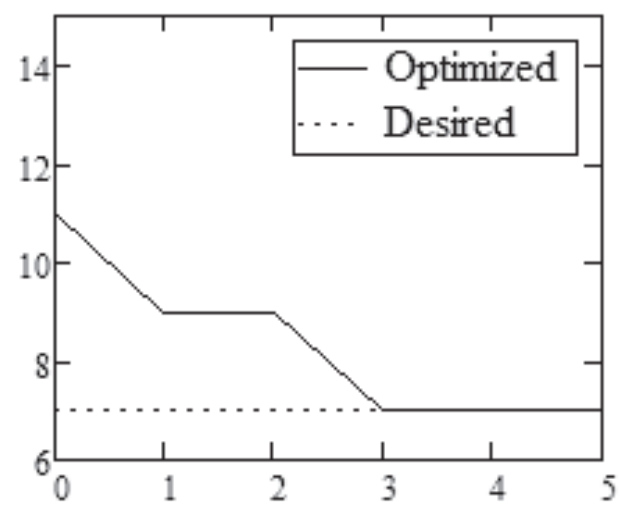

Class 8

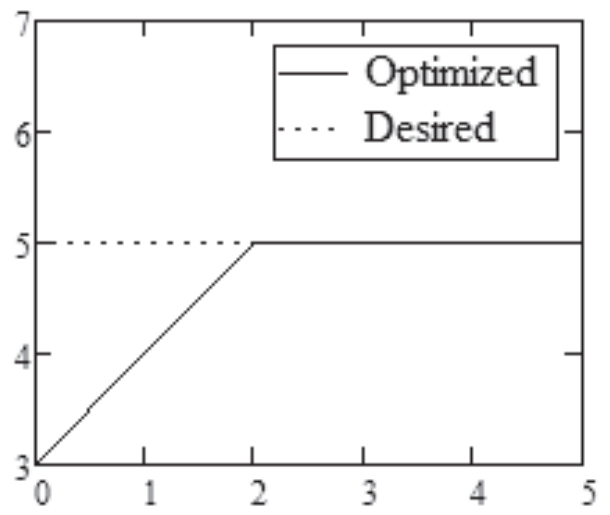

Figure 8. Classes 5-8, second scenario

Figure 9 shows the transition and fluctuation coefficients achieved by the algorithm.

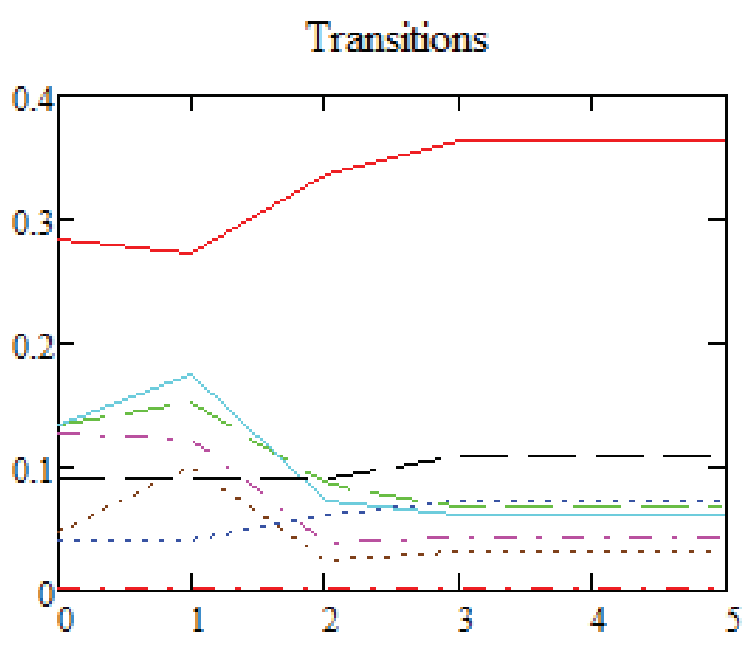




\section{Fluctuations}

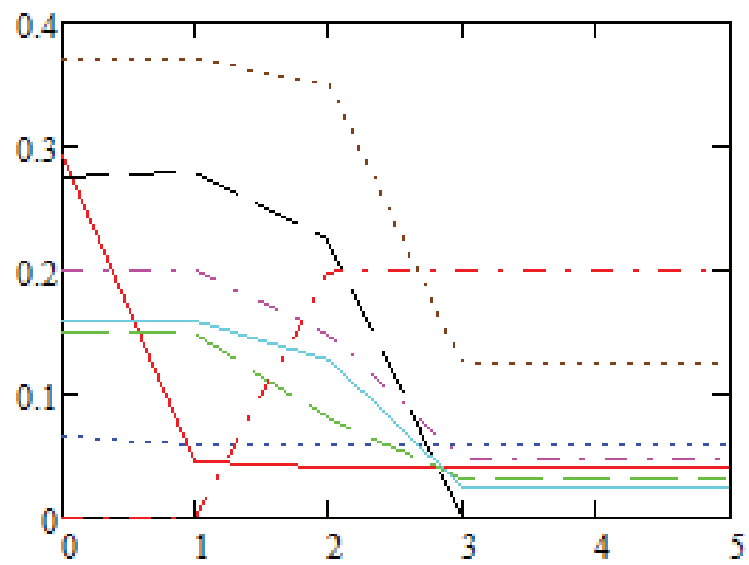

Figure 9. Transition and fluctuation coefficients, second scenario

The last step shows that the transition and fluctuation values are stable, as is the number of people in each rank. In Figure 10, the values of transitions and fluctuations are presented.

\section{Transitions}

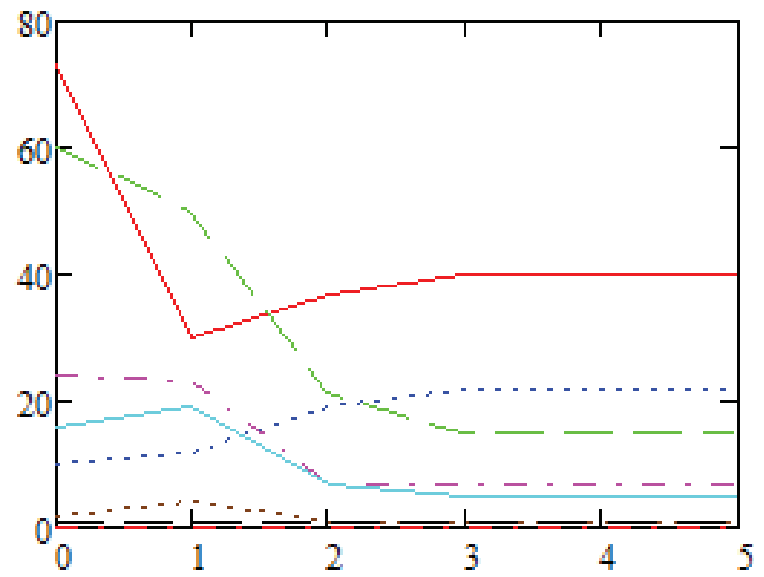

\section{Fluctuations}

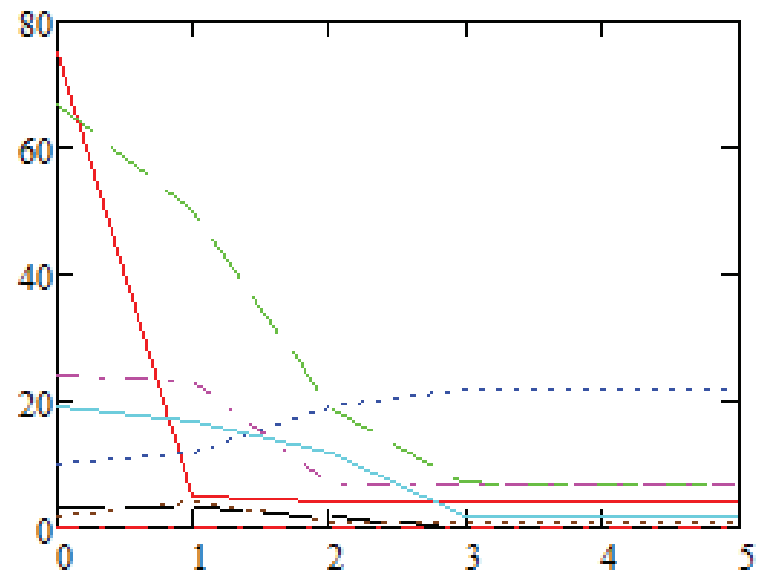

Figure 10. Transition and fluctuation values, second scenario

Although the transition coefficients have peaks, they cannot be considered as oscillations. Moreover, oscillations in the transition and fluctuation coefficients do not always result in non-monotonic transition values.

For the third scenario, the following results were achieved. The recruitment level change for the first class is shown in Figure 11. For this case, the algorithm requires eight steps to achieve the goal.

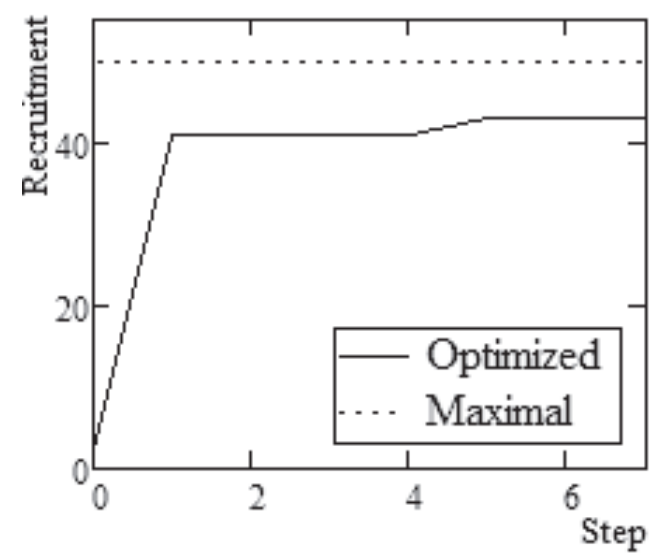

Figure 11. Recruitment level, third scenario

The recruitment level gradually rises but does not achieve the maximal possible level. In Figures 12 and 13, the change of the number of people in each class is shown. At time step 0 , the initial values are shown. For classes 1 and 2 , the goal is achieved in one step, while the other classes require more steps. For class 8 , the initial value is equal to the desired one, and the algorithm keeps it unchanged. 

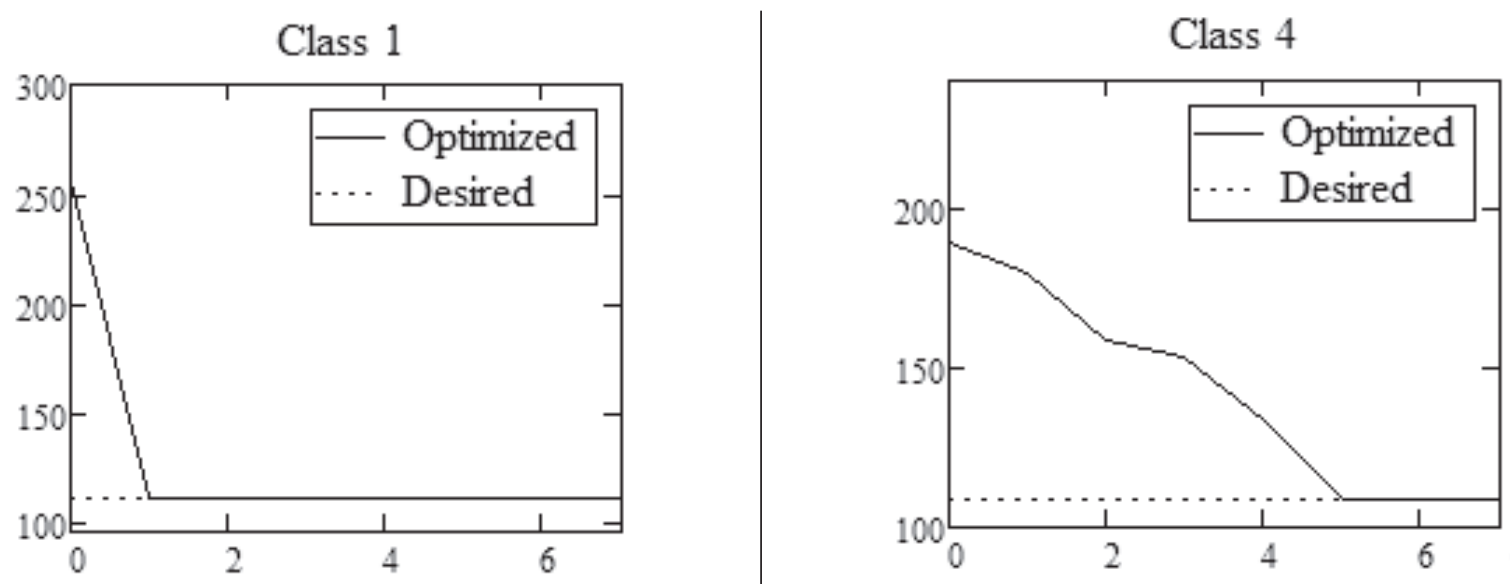

Class 2

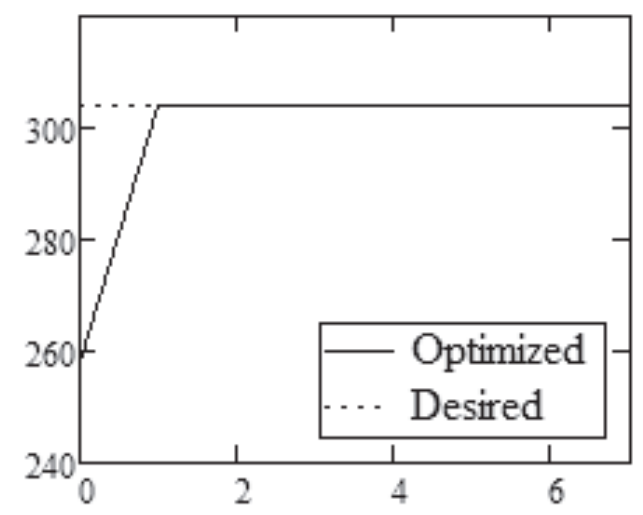

Class 3

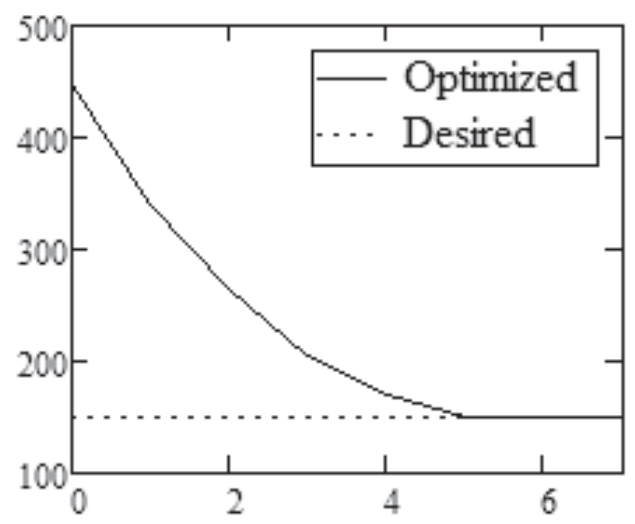

Figure 12. Classes 1-4, third scenario
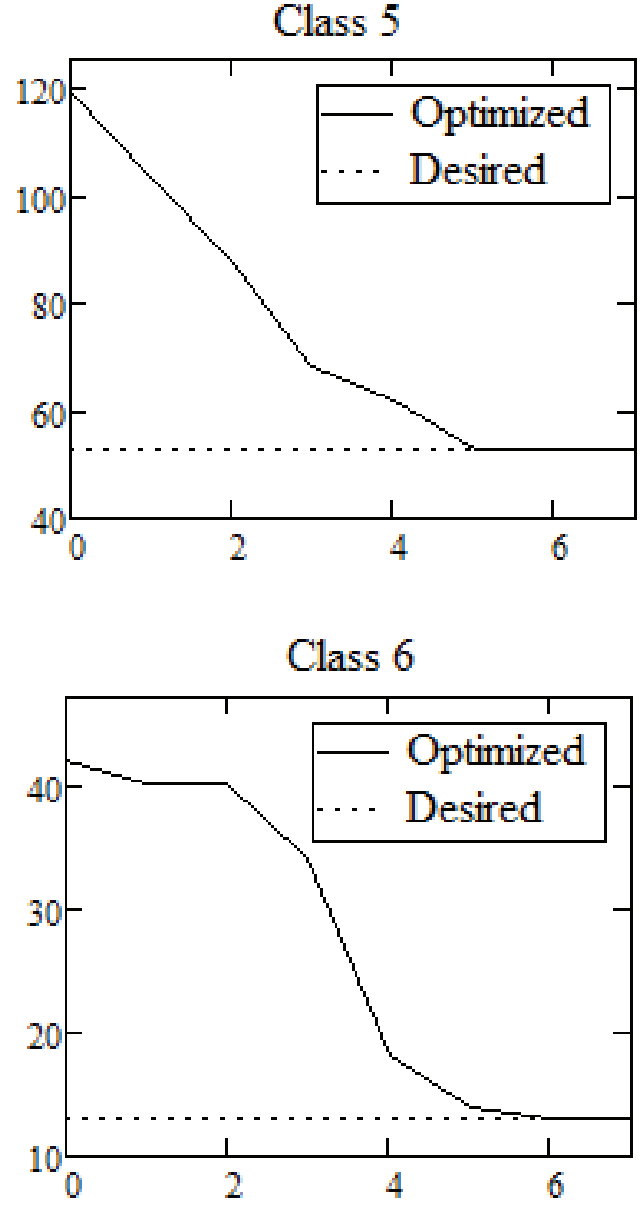


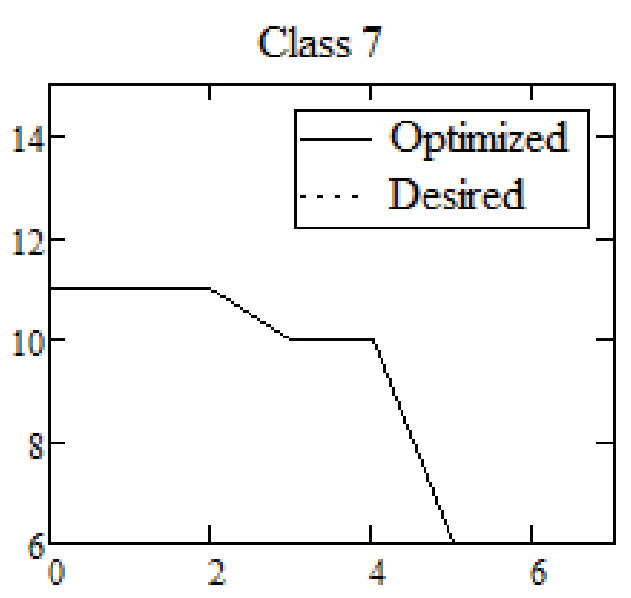

Class 8

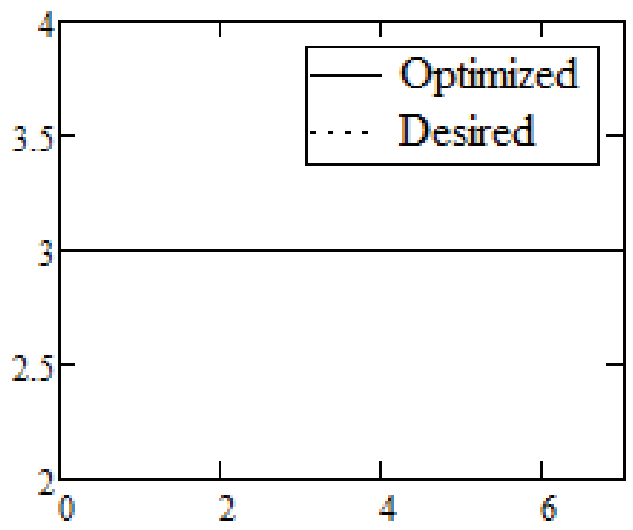

Figure 13. Classes 5-8, third scenario

Figure 14 shows the transition and fluctuation coefficients achieved by the algorithm.

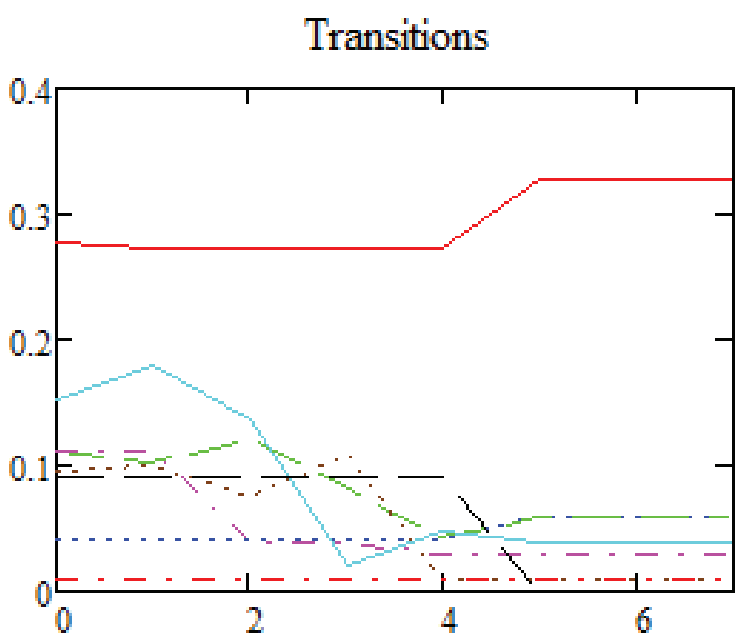

\section{Fluctuations}

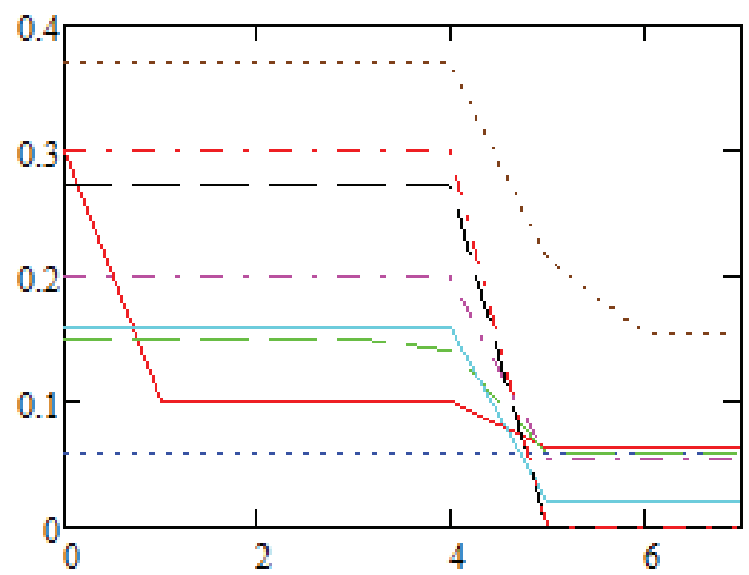

Figure 14. Transition and fluctuation coefficients, third scenario

The last step shows that the transition and fluctuation values are stable, as are the number of people in each class. In Figure 15 the values of transitions and fluctuations are presented.

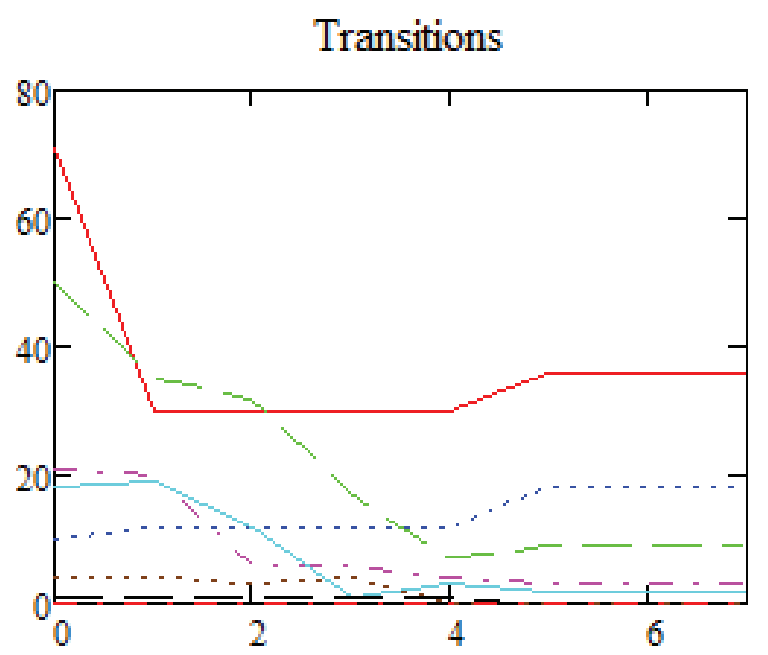




\section{Fluctuations}

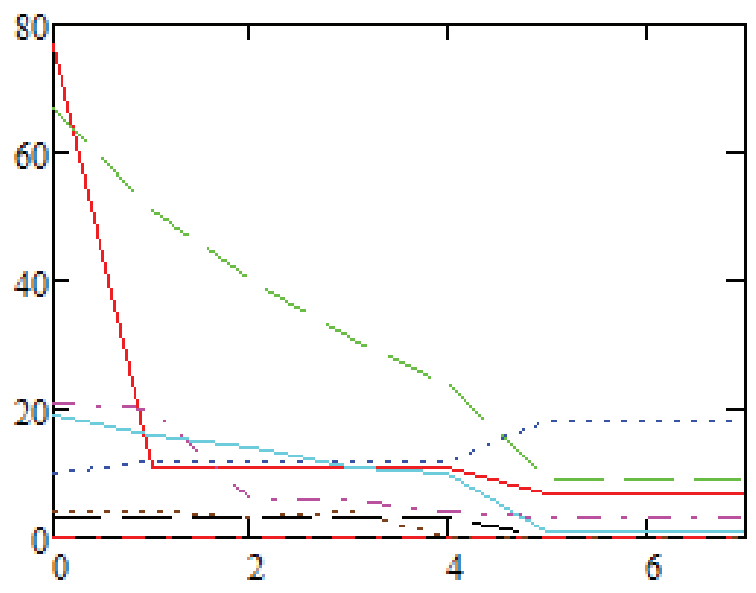

Figure 15. Transition and fluctuation values, third scenario

As with the previous scenario, the peaks in transition coefficients may be observed; however, these peaks are allowable as they are not oscillations.

The presented results show that the proposed method allows finding optimal solutions for different scenarios of human resource planning problems. Testing the algorithm on a set of 25 other scenarios with randomly generated parameters has shown similar results, proving the algorithm's efficiency.

\section{Conclusion}

The applied mathematical model and developed optimization algorithm provided the solutions to the problem of finding proper transition strategies in the strict hierarchical human resources management system. With the proper strategy, a short time is needed to achieve new numbers in particular classes without oscillations in transition and wastages. The task of providing the proper strategies is demanding, since the problem is dynamic, meaning that each parameter of the model could be changed in time. This generates a large search space, which is challenging for the optimization algorithm. Another challenge is the possibility to provide two equally good strategies with different control vectors. This means that the optimum criterion is not fully defined. Nevertheless, the developed, advanced local stochastic search algorithm provided proper solutions, eliminating the oscillations, achieving the target values in the shortest possible time.

\section{Acknowledgment}

This research was supported by the Ministry of Higher Education, Science and Technology of the Republic of Slovenia, (Contract No.: BI-RU/14-15-047, Contract No.
P5-0018) and Russian Federation Presidential Scholarship No. 16-in-689.

\section{Literature}

Babaei, M., Zahra, G., \& Soudabeh, A. (2015). Challenges of Enterprise Resource Planning implementation in Iran large organizations. Information Systems, 54, 15-27, http://dx.doi. org/10.1016/j.is.2015.05.003

Borštnar Kljajić, M., Kljajić, M., Škraba, A., Kofjač, D., \& Rajkovič, V. (2011). The relevance of facilitation in group decision making supported by a simulation model. System Dynamics Review, 27(3), 270-293, http://dx.doi.org/10.1002/ sdr.460

Cabanillas, C., Resinas, M., del-Río-Ortega, A., \& Cortés, A.R. (2015). Specification and automated design-time analysis of the business process human resource perspective. Information Systems, 52, 55-82, http://dx.doi.org/10.1016/j.is.2015.03.002

Gupta, A., \& Ghosal, A. (2014). A manpower planning model based on length of service under varying class sizes. OPSEARCH, 51(4), 615-623, http://dx.doi.org/10.1007/s12597-013-0162-1

Kljajić M., Bernik I., \& Škraba A. (2000). Simulation approach to decision assessment in enterprises. Simulation, 75 (4), 199210, http://dx.doi.org/10.1177/003754970007500402

Kofjač, D., Bavec, B., \& Škraba, A. (2015). Web application for hierarchical organizational structure optimization - human resource management case study. Organizacija, 48(3), 177187, http://dx.doi.org/10.1515/orga-2015-0012

Lin, D., Yue, T., Ganggang, N., Yongqing, X., Xin, S., Changrui, R., \& Zongying, Z. (2015). Scheduling Workforce for Retail Stores with Employee Preferences. In 2015 IEEE International Conference on Service Operations And Logistics, And Informatics (SOLI), 15-17 November 2015 (pp. 37-42). Piscataway, NJ, USA: IEEE.

Majd, A. M. K., Mahootchi, M., \& Zakery A. (2015). A reinforcement learning methodology for a human resource planning problem considering knowledge-based promotion. Simulation Modelling Practice and Theory, in press, http://dx.doi.org/ 10.1016/j.simpat.2015.07.004

Rozman, Č., Pažek, K., Kljajić, M., Bavec, M., Turk, J., Bavec, F., Kofjač, D., \& Škraba, A. (2013). The dynamic simulation of organic farming development scenarios-A case study in Slovenia. Computers and Electronics in Agriculture, 96, 163172, http://dx.doi.org/10.1016/j.compag.2013.05.005

Škraba, A, Kljajić, M-, Papler, P., Kofjač, D., \& Obed, M. (2011). Determination of recruitment and transition strategies. Kybernetes, 40(9/10), 1503-1522, http://dx.doi. org/10.1108/03684921111169512

Škraba A., Kljajić M., \& Kljajić, M.B. (2007). The role of information feedback in the management group decision-making process applying system dynamics models. Group Decision and Negotiation, 16(1), 77-95, http://dx.doi.org/10.1007/ s10726-006-9035-9

Škraba A., Kljajić M., \& Leskovar R. (2003). Group exploration of system dynamics models - is there a place for a feedback loop in the decision process? System Dynamics Review, 19(3), 243-263, http://dx.doi.org/10.1002/sdr.274

Škraba, A., Kofjač, D., Žnidaršič, A., Maletič, M., Rozman, Č., Semenkin, E.S., Semenkina, M. E., \& Stanovov, V. V. (2015). 
Application of Self-Configuring genetic algorithm for human resource management. Journal of Siberian Federal University - Mathematics and Physics. 8(1), 94-103.

Škraba, A., Kofjač, D., Žnidaršič, A., Rozman, Č., \& Maletič, M. (2015). Application of finite automata with genetic algorithms in JavaScript for determination of manpower system control. Vestnik Sibirskogo gosudarstvennogo aèrokosmičeskogo universiteta imeni M. F. Rešetneva, 16(1), 153-158.

Škraba, A., Stanovov, V. V., Žnidaršič, A., Rozman, Č., \& Kofjač, D. (2015). Consideration of optimal control of strictly hierarchical manpower system. In The $4^{\text {th }}$ International Workshop on Mathematical Models and their Applications IWMMA 2015, 24-26 November 2015 (p. 9), Krasnoyarsk, Russia: Siberian State Aerospace University.

Andrej Škraba obtained his Ph.D. in the field of Organizational Sciences - Informatics from the University of Maribor. He works as an associate professor and researcher in the Cybernetics \& Decision Support Systems Laboratory at the Faculty of Organizational Sciences, University of Maribor. His research interests cover systems theory, modeling and simulation, cyberphysical systems and decision processes. He is a member of System Dynamics Society and SLOSIM.

Vladimir Stanovov received the B.S. and M.S. degrees in system analysis and control from Reshetnev Siberian State Aerospace University, Krasnoyarsk, Russia, in 2012 and 2014, respectively. His research interests include genetic fuzzy systems, self-configured evolutionary algorithms and machine learning. He is currently a PhD student at the Siberian State Aerospace University.
Mr. Stanovov received the Best Student Paper Award from the 4th International Congress on Advanced Applied Informatics in 2015.

Eugene Semenkin received his Master in Applied Mathematics degree from Kemerovo State University (Kemerovo, USSR) in 1982, his PhD in Computer Science from Leningrad State University (Leningrad, USSR) in 1989 and his DSc in Engineering and Habilitation from the Siberian State Aerospace University (Krasnoyarsk, Russia) in 1997. Since 1997, he has been a professor of systems analysis at the Institute of Computer Science and Telecommunications of the Siberian State Aerospace University. His areas of research include the modelling and optimization of complex systems, computational intelligence and data mining. He has been awarded the Tsiolkovsky Badge of Honour by the Russian Federal Space Agency and the Reshetnev medal by the Russian Federation of Cosmonautics.

Davorin Kofjač obtained his Ph.D. from the University of Maribor in the field of Information systems management. He is a researcher and an assistant professor at the University of Maribor, Faculty of Organizational Sciences in the Cybernetics and Decision Support Systems Laboratory. His main research interests include modelling and simulation, decision support systems, operational research and artificial intelligence. He was involved in many EU, NATO, bilateral and national projects and is an author of more than 120 publications in international journals, monographs and conferences. He is a member of ACM, INFORMS and SLOSIM. 